\title{
Concepto de objetivo de una tarea matemática de futuros maestros
}

\section{The Objective Concept of a Mathematical Task for Future Teachers}

\author{
Carmen Gloria Aguayo-Arriagada* \\ ORCID iD 0000-0001-9576-2312 \\ Pablo Flores ${ }^{* *}$ \\ ORCID iD 0000-0002-3292-6639 \\ Antonio Moreno*** \\ ORCID iD 0000-0002-8284-3903
}

\begin{abstract}
Resumen
Dentro de la línea de formación de profesores, en este trabajo presentamos aspectos de una investigación con futuros maestros de primaria, donde nos enfocamos en comprender cómo los estudiantes de la Universidad de Granada interpretan la meta como elemento de análisis de una tarea matemática escolar, enmarcada dentro del Análisis Didáctico como herramienta funcional en la formación inicial. Se ha seguido una metodología cualitativa, a través del análisis de contenido. Los antecedentes evidencian la importancia de las tareas escolares para favorecer el aprendizaje de las matemáticas, y los resultados nos muestran la dificultad que presentan los futuros maestros para establecer y definir la meta de una tarea matemática escolar.
\end{abstract}

Palabras clave: Tarea matemática. Objetivo. Formación de maestros. Análisis Didáctico.

\begin{abstract}
Within the line of teacher training, we present in this work aspects of a research with future elementary school teachers, where we focus on understanding how students of the University of Granada interpret the objective as an analysis element of a school mathematical task, framed within the Didactic Analysis as a functional tool in the initial formation. A qualitative methodology has been followed through content analysis. The antecedents show the importance of the school tasks to favor mathematics learning and the results show us the difficulty that the future teachers present to establish and to define the objective of a school mathematical task.
\end{abstract}

Keywords: School Mathematical Task. Objective. Teacher Training. Didactic Analysis.

\section{Introducción}

\footnotetext{
* Máster en Didáctica de la Matemática por la Universidad de Granada (UGR), España. Doctoranda en Ciencias de la Educación de la Universidad de Granada (UGR), Granada, España. Dirección postal: Campus de Cartuja, s/n, Granada, España, C.P: 18071. E-mail: carmenaguayo@ correo.ugr.es.

${ }^{* *}$ Doctor en Matemáticas por la Universidad de Granada. Profesor Titular de la Universidad de Granada (UGR), Granada, España. Dirección postal: La Cartuja, s/n, Granada, España, C.P: 18011. E-mail: pflores@ugr.es.

*** Doctor en Didáctica de las Matemáticas por la Universidad de Granada. Profesor de la Universidad de Granada (UGR), Granada España. Dirección postal: La Cartuja, s/n, Granada, España, C.P: 18011. E-mail: amverdejo@ugr.es.
} 
Uno de los problemas de investigación en la formación de profesores de matemáticas, es examinar modelos de planes de formación (PONTE; CHAPMAN, 2008). Uno de los tomos del pasado handbook sobre el profesor de matemáticas se dedica a las herramientas para la formación de profesores (TIROSH; WOODS, 2008), especialmente los casos, las tareas y las investigaciones. Ponte y Chapman (2008), resumiendo aportes de diversos programas de formación, destacan, también, el uso de actividades (tareas) matemáticas escolares, junto al análisis, resolución y enriquecimiento de problemas y actividades de investigación.

En estos documentos apreciamos que las tareas son vistas bajo dos perspectivas: como tarea matemática escolar, base para el aprendizaje de los alumnos (DOYLE, 1988), y la tarea formativa profesional, que se utiliza en la formación de profesores (TIROSH; WOODS, 2008). Una de las capacidades clave que debe desarrollar el maestro en formación para adquirir la competencia profesional de planificar, incluye diseñar tareas matemáticas escolares y organizarlas en secuencias didácticas (RICO, 2016).

Apreciar el papel de las tareas matemáticas escolares en la enseñanza (DOYLE, 1988) identificando sus componentes de manera crítica (WATSON; MASON, 2007), lleva a Liljedahl, Chernoff y Zazkis (2007) a proponer un proceso de selección y mejoramiento de tareas que consta en cuatro fases: análisis predictivo, ensayo, análisis reflexivo y fase de ajuste. Este proceso se convierte en componentes de las tareas formativas profesionales.

Tomando como base estos principios, este trabajo, centrado en un programa de formación inicial de maestros en el área de matemáticas con un enfoque funcional, examina cómo llevan a cabo el análisis de tareas matemáticas escolares los futuros maestros, empleando para ello elementos del Análisis Didáctico (RICO, 2016).

Sullivan, Clarke y Clarke (2013) sostienen que puede aumentar el aprendizaje a través de las tareas matemáticas escolares, si el profesor tiene claridad en el foco matemático y en los fines pretendidos con su utilización. Moreno y Ramírez-Uclés (2016) consideran que el análisis de una tarea matemática consiste en identificar, entre otras componentes, la meta, su expectativa de aprendizaje.

Conseguir que futuros profesores identifiquen objetivos de aprendizaje es una de las intenciones formativas de los cursos de formación. En este artículo analizamos cómo futuros maestros identifican y formulan la meta de una tarea matemática escolar, dentro de un módulo basado en el análisis de tareas matemáticas escolares, donde una tarea formativa consiste en analizar una tarea matemática escolar, el bricolaje (ver Anexo 1), elaborada por los formadores. Recogemos las producciones de los estudiantes y examinamos qué significa para ellos la meta de la tarea. 
En el artículo comenzamos por presentar la tarea matemática escolar y de formación, el objetivo de aprendizaje (meta de la tarea), elementos del análisis didáctico empleados en el curso formativo y en la investigación (RICO, 2015). Posteriormente, describimos la metodología, presentamos los resultados y las conclusiones.

\section{Formación inicial de profesores de matemáticas}

Para introducirnos en el tema de la formación inicial de maestros de primaria es necesario concretar el conocimiento que estos deben adquirir. Desde la separación de Shulman (1986) entre conocimiento del contenido y conocimiento didáctico del contenido, se ha atendido al conocimiento profesional del profesor de matemáticas (SULLIVAN; WOODS, 2008).

En nuestra investigación nos basamos en el conocimiento del profesor que plantea Rico (2015), quien presenta el Análisis Didáctico como una componente funcional, que establece el conocimiento del contenido matemático escolar derivado del análisis de contenido y el conocimiento didáctico de las matemáticas escolares derivado de los análisis cognitivo, de instrucción y de actuación. El primero es planteado como el conjunto de saberes sobre un contenido matemático específico, que se establece en base a tres componentes de significado: definición - representación - sentido.

El conocimiento didáctico de las matemáticas escolares es el conjunto de conocimientos teóricos, técnicos y prácticos sobre la enseñanza y aprendizaje de las matemáticas escolares (RICO, 2015). Esta organización del conocimiento se refleja en el curso que analizamos, que tiene como contenidos las herramientas didácticas necesarias para profundizar en el significado del contenido (análisis de contenido), en aspectos cognitivos del aprendizaje (análisis cognitivo, expectativas, limitaciones y oportunidades de aprendizaje), y en la instrucción (tareas matemáticas escolares), herramientas necesarias para planificar la enseñanza de un contenido matemático (RICO, 2015).

La profundización en el conocimiento matemático, buscando el significado del contenido matemático, su relación con el currículo y disponer de herramientas para iniciarse en el conocimiento didáctico del contenido, es la idea que fundamenta el programa de formación en el Grado de Educación Primaria de la Universidad Granada, con la intención de enfatizar la dimensión profesional del futuro profesor (FLORES, 2013).

Estas ideas arrancan de los trabajos de Rico (1997a, 1997b) para diseñar la formación inicial de maestros en el área de matemática basándose en tres principios (FLORES, 2013): favorecer la comprensión de los conceptos matemáticos; facilitar acceso a lecturas y resultados 
de didácticas de los temas matemáticos; hacer que el conocimiento adquiera un papel práctico, estimulando a que profundice en el significado de los contenidos matemáticos para diseñar sesiones de clase encaminadas a lograr su aprendizaje.

Rico (1997a, 1997b) establece dimensiones sobre las que reflexionar al planificar clases, concretadas en Organizadores del Currículo. Los organizadores del currículo generan el Análisis Didáctico (RICO, 2015) que constituye una herramienta para el profesor, para el diseño, implementación y evaluación de la enseñanza y aprendizaje de un tema de matemáticas. Los elementos del Análisis Didáctico se convierten en contenidos del curso de formación, planteados de manera funcional, pues son herramientas para construir conocimiento didáctico de las matemáticas escolares.

El Análisis Didáctico está estructurado en cuatro: Análisis de Contenido, Análisis Cognitivo, Análisis de Instrucción y Análisis de Evaluativo (RICO, 2015). La formación comienza trabajando el significado de los contenidos de las matemáticas de Educación Primaria, atendiendo a la terna, concepto, representación y sentido, en el primer año de carrera. Posteriormente, se presentan herramientas para que los estudiantes realicen los cuatro análisis mencionados, examinando aspectos cognitivos e instructivos (tareas matemáticas escolares, secuencias de enseñanza y planificación de la evaluación) (FLORES, 2013), que apuntan al desarrollo del conocimiento didáctico de las matemáticas escolares, en asignaturas del segundo, tercer y cuarto año de carrera. Esto muestra la relación y coherencia entre el plan de formación inicial de futuros maestros de primaria de la Universidad de Granada y el desarrollo de sus conocimientos para la planificación del proceso de enseñanza y aprendizaje sobre un contenido matemático escolar.

\section{Tareas Matemáticas}

La enseñanza de las matemáticas se organiza a partir de actividades del estudiante que responden a tareas matemáticas (DOYLE, 1988). Empleamos el término tarea para designar las acciones instructivas estructuradas, referidas a un contenido matemático, que establecen las demandas matemáticas, y la gestión e interacción prevista. Watson et al. (2013, p. 12) consideran que:

[...] la tarea genera actividad con la que aflora la oportunidad para encontrar conceptos matemáticos, ideas, estrategias y para usar y desarrollar pensamiento matemático y formas de interrogarse [..], la enseñanza incluye la selección, modificación, diseño, secuenciación, instalación, observación y evaluación de tareas.

Por tanto, uno de los conocimientos que debe adquirir el futuro maestro de primaria en 
su formación inicial para la planificación de unidades didácticas, es el de tarea matemática (eje central de nuestra investigación) (SHIMIZU et al., 2010).

Los cursos de formación de profesores de matemáticas también están constituidos por tareas formativas, algunas de las cuales tendrán por objetivo aprender a planificar clases, es decir, diseñar secuencias de tareas matemáticas escolares. Es, por tanto, necesario hacer la distinción entre tareas matemáticas escolares y tareas de formación (TIROSH; WOODS, 2008), diferenciadas por los agentes a quien están dirigidas y los sistemas educativos en que se plantean.

\subsection{Tareas matemáticas escolares (TME)}

Para Stein y Smith (1998, p. 268) "una tarea se define como un segmento de la actividad de clase que se dedica al desarrollo de una idea matemática particular". Investigaciones pioneras sobre TME son las desarrolladas por Doyle (1988), estudiando la enseñanza examinando la naturaleza de las tareas implementadas por el profesor.

Sullivan et al. (2010) comparan tres profesores que implementaron la misma tarea en clase, apreciando cómo las actividades reflejan sus intenciones, y concluyen señalando que la aplicación de una TME está influenciada por la capacidad del profesor para modificarla, según su conocimiento matemático y la claridad de los objetivos que pretende lograr.

Otras investigaciones destacan la importancia de que el profesor tenga claros los objetivos que pretende lograr cuando planifica, ya que la TME no es suficiente para garantizar el aprendizaje de los estudiantes (SULLIVAN; CLARKE; CLARKE, 2013). Es necesario que el profesor defina, con claridad, los objetivos en su planificación.

Schunk (1996) enfatiza que las metas que incorporan estándares específicos de desempeño son más propensas a mejorar el aprendizaje que los objetivos más generales. La revisión bibliográfica realizada muestra diferencias entre investigadores, mientras unos hablan de objetivo de la tarea, otros lo llaman meta.

Para aclarar la diferenciación nos basamos en los Niveles de objetivos en el currículo de Rico y Lupiáñez (2008) quienes establecen que, según el ámbito (etapa, curso o tema), se generan diferentes finalidades. En este trabajo partimos de considerar las metas como objetivos específicos, es decir “aquellos que trabajan unos determinados y precisos contenidos, requieren de unas capacidades para atender a unas tareas y se identifican por ciertas conductas que el estudiante debe manifestar" (FLORES; LUPIÁÑEZ, 2016, p.184).

Por tanto, la meta de una TME es el propósito que el profesor asigna a una tarea. 
Teniendo en cuenta que el planificar una unidad didáctica arranca de los objetivos planteados en el currículo para establecer las expectativas de aprendizaje, una TME tiene que clarificar su finalidad, ayudando esto a determinar su meta para definir específicamente lo que se quiere lograr con la misma.

\subsection{Tareas de formación}

Definida la meta de una TME como variable de estudio en este trabajo, justificamos su relevancia conjeturando que la formación inicial tiene que suministrar herramientas funcionales a los futuros maestros para elaborar unidades didácticas, con bases sólidas sobre el conocimiento del contenido matemático escolar (análisis de contenido) y el conocimiento didáctico de las matemáticas escolares (análisis cognitivo, de instrucción y evaluativo). En este proceso de formación se plantean tareas formativas, entre ellas el análisis de TME.

Investigaciones previas sobre este tema son las de Arbaugh y Brown (2005), que trabajaron con un grupo de siete profesores que analizaron tareas matemáticas según Niveles de Demanda Cognitiva, examinando cómo influyó en su selección para el aula, concluyendo que los profesores mejoraron su forma de considerar las TME, por lo que resaltan la importancia de involucrar a los maestros en analizar tareas de manera crítica.

Basándonos en las ideas de Watson y Mason (2007) de que las tareas matemáticas de formación comparten mucho de las TME, las tareas de los futuros maestros tienen propósitos más ambiciosos, ya que enfatizan el contenido matemático y a su vez cuestiones de didáctica de la matemática (LILJEDAHL; CHERNOFF; ZAZKIS, 2007).

Para relacionar el conocimiento matemático y el conocimiento didáctico en torno a una TME, en el Grado de primaria de la Universidad de Granada se plantea una tarea formativa que demanda el análisis de una TME. Watson y Mason (2007) hicieron una revisión de varios programas de formación en todo el mundo, apreciando que algunos promovían el pensamiento crítico de los futuros maestros sobre tareas, analizando libros de texto, inventandolas, probándolas y evaluándolas. Liljedahl, Chernoff y Zazkis (2007) plantean diseño de TME en formación inicial por creación de tareas nuevas o por adaptación de tareas ya existentes, plantean un proceso de cuatro etapas: análisis predictivo, ensayo, análisis reflexivo y ajuste.

El proceso que plantean Liljedahl, Chernoff y Zazkis (2007) tiene bastantes similitudes con el que se desarrolla en el curso Diseño y Desarrollo del Currículo de Matemática en la Educación Primaria que se imparte en el tercer año del Grado de la Universidad de Granada, donde a los futuros maestros se les presenta una tarea matemática escolar, la resuelven como 
estudiantes para, posteriormente, hacer un análisis de dicha tarea, teniendo en cuenta diferentes elementos (meta, formulación, materiales y recursos, agrupamiento, interacción y temporalidad).

\section{Objetivos}

Las investigaciones reseñadas concuerdan en que un elemento primordial de la tarea es su función, es decir, identificar el aprendizaje que se quiere alcanzar mediante ella.

Siguiendo a Gómez y Romero (2015, p. 69), empleado como texto en el curso formativo, llamamos meta a la finalidad cognitiva de la tarea, definida por estos autores como el "propósito que el profesor asigna a una tarea, los objetivos de aprendizaje a los que la tarea pretende contribuir y los errores y dificultades que espera que la tarea contribuya a superar".

Por tanto, el concepto de meta como expectativa nos lleva al de objetivo, término que aparece en la enseñanza en la mitad del siglo XX, arrancando de la aplicación de la teoría de sistemas a la enseñanza, en vista a una tecnificación de la misma. En una primera época incorpora una visión conductual del aprendizaje, señalando conductas finales observables y medibles.

Pese a la crítica a esta visión simplista de la enseñanza como sistema, la concepción constructivista del aprendizaje mantiene a los objetivos como un elemento de la programación para dar racionalidad al proceso instructivo (FERNÁNDEZ PÉREZ; GIMENO; ZABALZA, 1977), perdiendo de vista la intención de operativizar finalidades nucleares, pasando a seleccionar expectativas de aprendizaje con dos funciones: servir de guía a los contenidos y a las actividades de aprendizaje y proporcionar criterios para el control de esas actividades (PÉREZ-GARCÍA, 2010).

La forma en que se formulan y entienden las intenciones de aprendizaje puede variar, por lo que distinguimos objetivos de instrucción (o de enseñanza) y objetivos de aprendizaje, que difieren en el sujeto que se ve afectado por ellos. Los primeros indican intenciones docentes (iniciar un tema, introducir a los estudiantes en, facilitar el contacto con...) o bien describen una forma de hacer (trabajen en grupo, por ejemplo). Los objetivos de aprendizaje describen lo que se espera que los alumnos adquieran como consecuencia de la realización de la tarea, para lo que tienen que atender a la especificidad, premeditación, deliberación, indivisibilidad cognitiva y compatibilidad (DELONG; WINTER; YACKEL, 2005). El tipo de formulación que tienen los objetivos planteados por los futuros maestros es la primera variable de nuestro estudio, con las dos categorías señaladas (objetivos de instrucción, objetivos de aprendizaje). 
Definir el objetivo no es una labor fácil para el profesor, pues requiere una visión global de las expectativas de aprendizaje en los diferentes niveles curriculares. Rico y Lupiañez (2008) asumen que un objetivo requiere tres componentes: la dimensión cognitiva, que corresponde a las capacidades que expresan actuación y que se identifican por uno o varios verbos de acción; la dimensión cultural o contenido matemático; y la dimensión práctica, que se plasma al expresar el contexto de aplicación. Esta estructura del objetivo determina el segundo aspecto que estudiamos, los elementos del objetivo, que determinan tres variables de nuestro estudio: capacidades, contenidos y contextos.

Esta forma de organizar el concepto de objetivo nos lleva a emplear los elementos considerados en el Análisis Didáctico como dimensiones para apreciar la riqueza de elementos que incorporan los estudiantes cuando construyen objetivos específicos (metas).

Formular objetivos de aprendizaje es una tarea compleja. DeLong, Winter y Yackel (2005) reúnen diversas investigaciones que encontraron discrepancias entre los objetivos formulados por profesores y su práctica de enseñanza. Ruiz-Hidalgo et al. (2017) examinan el conocimiento didáctico sobre objetivos de futuros maestros, analizando el aprendizaje que consideraban que alcanzarían los alumnos que resolvieran una demanda matemática simple, un ejercicio (FERREIRA; BURIASCO, 2015): representa 2/3.

Los autores aprecian la disposición de los estudiantes a realizar una redacción formal de los objetivos, incluyendo aspectos generales, o incorporando capacidades genéricas poco precisas, mostrando dificultades para diferenciar objetivos de aprendizaje de objetivos de enseñanza. Santos, Ortigão y Aguiar (2014) examinan las expectativas de aprendizaje que identifican profesores de matemáticas de los primeros años de primaria, apreciando que seleccionan, preferentemente, las que se derivan de los libros de texto, especialmente las que corresponden a habilidades más simples.

\section{Objetivos de investigación}

En la formación de futuros maestros de primaria recurrimos a una tarea de formación consistente en analizar una TME, siendo el primer paso la identificación de la meta. En estas circunstancias, nos planteamos la siguiente pregunta general de investigación: ¿Cómo ponen en juego el conocimiento tratado en la formación inicial los futuros maestros de primaria, cuando planifican unidades didácticas? En este trabajo empezamos por apreciar cómo los futuros maestros de primaria de la Universidad de Granada identifican, establecen e interpretan la meta de una TME, cuando llevan a cabo el análisis de la misma. Por lo tanto, nos planteamos como 
objetivos:

Objetivo 1. Describir los conocimientos didácticos sobre la meta de una tarea matemática escolar que ponen en juego futuros maestros de primaria.

Objetivo 2. Comprender qué interpretación dan futuros profesores de primaria a la meta de una tarea matemática escolar.

\section{Metodología}

Se trata de un estudio interpretativo, empleando una metodología cualitativa, teniendo en cuenta que este tipo de investigación se basa en una lógica y procesos inductivos (explorar y describir, y, luego, generar perspectivas teóricas). Al querer comprender el trabajo realizado por futuros profesores de primaria, se enmarca dentro de un estudio descriptivo (HERNÁNDEZ; FERNÁNDEZ; BAPTISTA, 2010).

\subsection{Contexto}

El estudio se enmarca en la asignatura Diseño y Desarrollo del Currículo de Matemática en Educación Primaria de los cursos académicos 2013/2014 y 2015/2016, del Grado en Educación Primaria de la Universidad de Granada. Esta asignatura tiene como

[...] principal finalidad que los estudiantes profundicen y apliquen su conocimiento de las matemáticas y de las principales dimensiones que aborda la Didáctica de la Matemática, para diseñar, fundamentar y defender una unidad didáctica sobre algún tema específico de las matemáticas de Educación Primaria (UNIVERSIDAD DE GRANADA, 2014, p. 2).

En el desarrollo del curso una de las tareas de formación fue el análisis de una TME llamada bricolaje, diseñada por profesores del Departamento de Didáctica de la Matemática de dicha Universidad, para el aprendizaje del concepto de fracción en $4^{\circ}$ curso de primaria (AGUAYO-ARRIAGADA et al., 2014). La tarea el bricolaje es una tarea contextualizada, que plantea un reto abierto que requiere la actuación de los estudiantes, por lo que podemos identificarla con lo que Ferreira y Buriasco (2015) llaman una situación problemática.

El análisis de la TME ha comenzado resolviendo la tarea los futuros maestros, actuación indispensable para que puedan establecer tanto los contenidos matemáticos que se trabajan, como las expectativas que se quieren lograr con ella (SULLIVAN; CLARKE; CLARKE, 2013). El análisis de la tarea consiste en describir los siguientes elementos: meta, formulación, materiales y recursos, agrupamiento, interacción y temporalidad. Los estudiantes la analizan 
tomando en cuenta las previsiones de actuación, en cuanto a las acciones que realizan y los conocimientos que ponen en juego, como también al logro de expectativas, superación de limitaciones, significatividad y complejidad (GÓMEZ; ROMERO, 2015).

Hemos pedido a expertos un análisis previo de la TME el bricolaje, quienes la consideran una tarea compleja, que pretende la iniciación formal de los estudiantes en el fraccionamiento y la asignación de notaciones fraccionarias a las porciones resultantes, todas ellas fracciones propias (AGUAYO-ARRIAGADA et al., 2014).

\subsection{Sujetos}

Los futuros maestros, estudiantes de los cursos 2013/2014 y 2015/2016, trabajaban en grupos, por lo que los sujetos son 35 grupos (17 grupos del curso 2013/2014 -grupos notados con minúscula g y 18 grupos del curso 2015/2016 -señalados con mayúscula, G).

\subsection{Instrumento}

Se utiliza como instrumento de recogida de datos el trabajo final que entregaron los estudiantes. Se cuenta con producciones escritas que elaboraron los 35 grupos de trabajo, como respuesta a la tarea de formación (descripción y análisis de la TME bricolaje). Para extraer información de estas producciones se realiza un análisis de contenido, teniendo en cuenta lo planteado por Cohen, Manion y Morrison (2011), empleando como unidades de información las frases completas que describen el objetivo, aunque para su categorización se pueden tomar varios términos para situar en categorías diferentes.

\subsection{Variables de análisis}

Consideramos dos aspectos que generan cuatro variables. El Cuadro 1 describe aspectos, variables y dimensiones con que organizamos las respuestas.

\begin{tabular}{|l|l|l|}
\hline \multicolumn{1}{|c|}{ Aspectos } & \multicolumn{1}{|c|}{ Variables } & \multicolumn{1}{c|}{ Categorías/Dimensiones } \\
\hline 1) Formulación de objetivo & & $\begin{array}{l}\text { Objetivo de instrucción } \\
\text { Objetivo de aprendizaje }\end{array}$ \\
\hline 2) Elementos del objetivo & 2.1. Aspecto cognitivo, capacidad & Capacidades que delimitan \\
\cline { 2 - 3 } & 2.2. Aspecto cultural, contenido & $\begin{array}{l}\text { Elementos de la estructura que aparecen } \\
\text { Formas de representación } \\
\text { Aspectos fenomenológicos }\end{array}$ \\
\cline { 2 - 3 } & 2.3. Aspecto práctico, situaciones & Situaciones \\
\hline
\end{tabular}

Cuadro 1 - Aspectos, variables y dimensiones con las que organizan las respuestas Fuente: Elaboración propia 
La primera variable que corresponde al aspecto 1, se refiere a la forma de la respuesta y examina cómo se redacta el objetivo, a través de dos categorías: se redacta en forma de intención del profesor, centrado en sus expectativas de acción (objetivo de instrucción); o bien el objetivo refleja el aprendizaje que espera del alumno, señalando cuál es ese aprendizaje (objetivo de aprendizaje).

\subsubsection{Variable Capacidad}

La dimensión cognitiva del objetivo indica qué tipo de aprendizaje espera el profesor lograr. Identificamos esta dimensión con el término capacidad. En matemáticas escolares utilizamos este término para referirnos a la actuación de un estudiante con respecto a cierto tipo de tarea (por ejemplo, los problemas de transformar una forma simbólica de la función cuadrática - la estándar - en otra - la canónica).

Los objetivos de aprendizaje sobre un tópico concreto se suelen enunciar en términos de capacidades (RICO; LUPIAÑEZ, 2008). Un individuo ha desarrollado una cierta capacidad cuando puede resolver tareas que la requieren. Por lo tanto las capacidades se caracterizan por ser específicas a un tema concreto; pueden incluir o involucrar otras capacidades; y están vinculadas a tipos de tareas.

La selección de capacidades puede enfatizar aprendizajes de carácter mecánico (términos, convenios, destrezas), pero también abarcar la comprensión (conceptos, principios, procedimientos), o la aplicación funcional de estos aprendizajes a una situación nueva en la que tiene sentido (competencia). Para entender cuáles son las dimensiones cognitivas de los objetivos planteados, recogemos todos los verbos de acción, señalando con ellos las capacidades destacadas. No establecimos categoría previas, solamente identificamos los verbos.

\subsubsection{Variable Contenido}

Rico (1997b) utiliza el triángulo semántico de Frege para establecer el significado de un concepto matemático escolar, proponiendo tres componentes, la estructura conceptual, los sistemas de representación y la fenomenología. La estructura conceptual incluye las relaciones del concepto con otros conceptos, atendiendo tanto a la estructura matemática de la que el concepto forma parte, como a la estructura matemática que dicho concepto configura. Los 
sistemas de representación son las diferentes maneras en que se puede representar el concepto y sus relaciones con otros conceptos. La fenomenología engloba fenómenos (contextos, situaciones y/o problemas) que dan sentido o utilizan el concepto.

La TME analizada está relacionada con un concepto matemático, por lo tanto, podríamos decir que ésta contribuye a trabajar algunos de los componentes del significado de un concepto matemático, así mismo la meta que se quiere lograr con la tarea tomará en cuenta dichos componentes. Por esta razón, para identificar la concepción de meta de la TME bricolaje, identificamos conceptos, sistemas de representación y sentido que mencionan los futuros maestros de primaria dentro de su formulación de la meta. Estas dimensiones caracterizan esta variable. La dimensión estructura conceptual, sin categorías previas, identifica todos aquellos conceptos matemáticos que se planteaban en las metas.

La dimensión sistemas de representación se organiza por los diferentes sistemas, desde el más intuitivo (representación física), al más abstracto (simbólico), pasando por los icónicos (gráfico). La dimensión fenomenológica cubre los diferentes significados que tiene el concepto de fracción, basándonos en las diferentes interpretaciones que da Kieren (1980): relación partetodo, cociente, operador y razón.

\subsubsection{Variable Situación}

Hemos considerado importante apreciar si las metas que plantean los futuros maestros hacen alusión a algún tipo de situación, entendiendo que las situaciones aportan sentido a los contenidos matemáticos. Siguiendo el marco del estudio PISA 2012, entendemos que la situación, en una tarea matemática, es un aspecto del mundo del individuo en el cual se aplica un concepto matemático (RUIZ-HIDALGO, 2016) y considera cuatro tipos de situaciones para analizar y categorizar las tareas.

Consideramos relevante percibir si en el enunciado de la meta de una tarea matemática los futuros maestros hacen mención a alguna situación para dar sentido al concepto trabajado. La TME el bricolaje está enmarcada en una situación laboral (tienen que realizar un catálogo para una tienda de bricolaje), a partir de ello los estudiantes realizan acciones para aprender el concepto de fracción. Por lo tanto, esperamos que al plantear la meta, expresen una relación con la funcionalidad del aprendizaje esperado, mostrando las situaciones donde se pueden aplicar.

Empleamos las mismas categorías del estudio PISA, identificando situaciones personales, laborales, sociales y científicas. Las situaciones personales apuntan a las actividades 
cotidianas y familiares de los escolares; las laborales se centran en el mundo del trabajo; las sociales se refieren a las situaciones locales, nacionales o globales de determinados aspectos del entorno y las situaciones científicas se relacionan con aspectos de la ciencia y tecnología (RUIZ-HIDALGO, 2016).

\section{Resultados}

De las 35 producciones seleccionamos el apartado correspondiente a la meta, copiado en una tabla para ir identificando fragmentos de respuestas que constituirían unidades de análisis, que luego relacionamos con las categorías de análisis establecidas. Apreciamos que la mayoría de los grupos plantean más de una meta, tomando todas para su análisis.

\subsection{Formulación del objetivo}

Los 35 grupos plantean sus metas como Objetivos de aprendizaje, solamente dos grupos plantean, además, una de sus metas como Objetivo de instrucción:

- G1 plantea como primera meta una intención del profesor, la segunda es un objetivo de aprendizaje (Figura 1).

\section{Meta de la tarea matemática: En este caso la meta de esta tarea es introducir las fracciones en primaria de una manera mucho mas practica que los ejemplos que ponen los libros típicos de un trozo de tarta, $\mathrm{y}$ aprender a utilizar los números fraccionarios para expresar parte de la unidad.}

Figura 1 - Meta del grupo G1

Fuente: producciones de los estudiantes

- G17 comienza planteando un objetivo de aprendizaje, pero también menciona la intención del profesor de introducir el concepto de fracción (Figura 2).

La meta que es uno de los elementos de la tarea que vamos a analizar seria que los alumnos consigan aplicar las fracciones a objetos cotidianos y relacionarlas con ellas, con el objetivo de conocer el concepto de fracción y saber aplicarlo a la vida cotidiana. Introducir el concepto de fracción y aprender a utilizar números fraccionarios para expresar partes de la unidad. La dificultad principal que se le presenta al niño es a la hora de relacionarlo.

Figura 2 - Meta del grupo G17

Fuente: Producciones de los estudiantes 


\subsection{Elementos del objetivo}

\subsubsection{Variable capacidad}

En las metas planteadas por los futuros maestros se pueden identificar diferentes verbos que apuntan al desarrollo de una capacidad. Organizamos los verbos, empleando las dimensiones de la capacidad que aparece en Rico y Lupiáñez (2008), según los cuales la capacidad se compone de tres dimensiones, el contenido, la acción instructiva y el aspecto cognitivo. Consideramos que la formulación de la capacidad se dirige hacia el vértice cognitivo cuando apunta a una capacidad cognitiva general (aprender, comprender, etc.).

Los verbos de acción que se refieren a acciones generales, que suelen estar relacionadas con las actividades propuestas en la tarea (instrucción). Por último, los verbos de contenido aluden a una capacidad específica referida al aprendizaje de algún aspecto del contenido matemático, la fracción, por lo que entendemos que las capacidades específicas de este tema son: fraccionar, representar y dividir. La Figura 3 muestra un fragmento de la tabla donde se organizaron los verbos según lo mencionado anteriormente. Es importante destacar que todos los grupos hacían mención a más de un verbo en el planteamiento de sus metas.

\begin{tabular}{llll}
\hline Grupos & Cognitiva & Contenido & Instrucción \\
\hline G1 & aprender & & Utilizar, expresar \\
G2 & Comprender & Fraccionar & \\
G3 & aprender & & trabajar \\
G4 & Aprender, comprender & Fraccionar & \\
G5 & Relacionar & Fraccionar, representar & Ordenar, asignar \\
G6 & Aprender, comprender & Fraccionar, representar & \\
\hline
\end{tabular}

Figura 3 - Fragmento de tabla donde se organizaron los verbos.

Fuente: elaboración propia

Para analizar la tabla 1 comenzamos por contar la cantidad de verbos en cada dimensión, para establecer tendencias.

Tabla 1 - Coincidencias de los usos de cada tipo de verbo

\begin{tabular}{llc}
\hline \multicolumn{1}{c}{ Capacidades } & \multicolumn{1}{c}{ Grupos } & Frecuencia \\
\hline Cognitivos & $\mathrm{G} 11, \mathrm{G} 18, \mathrm{~g} 3, \mathrm{~g} 4, \mathrm{~g} 14, \mathrm{~g} 16, \mathrm{~g} 17$ & 7 \\
instrucción & $\mathrm{G} 7, \mathrm{~g} 15$ & 2 \\
Cognitivo - contenido & $\mathrm{G} 2, \mathrm{G} 4, \mathrm{G} 6, \mathrm{G} 9$ & 4 \\
Instrucción - contenido & $\mathrm{g} 11$ & 1 \\
Cognitivo - instrucción & $\mathrm{G} 1, \mathrm{G} 3, \mathrm{G} 10, \mathrm{G} 16, \mathrm{G} 17, \mathrm{~g} 2, \mathrm{~g} 5, \mathrm{~g} 7, \mathrm{~g} 8, \mathrm{~g} 9, \mathrm{~g} 13$ & 11 \\
Cognitivo - contenido - instrucción & $\mathrm{G} 5, \mathrm{G} 8, \mathrm{G} 12, \mathrm{G} 13, \mathrm{G} 14, \mathrm{G} 15, \mathrm{~g} 1, \mathrm{~g} 6, \mathrm{~g} 10, \mathrm{~g} 12$ & 10 \\
\hline
\end{tabular}

Fuente: elaborado por los autores 
Los verbos cognitivos son el $41 \%$ del total de verbos, los de contenido un $18 \%$ y los verbos de instrucción un 44\%. Examinando por filas la tabla de la Figura 3, encontramos 6 tipos de coincidencias: 1) Grupos que en sus metas mencionan capacidades sólo cognitivas; 2) Grupos que en sus metas mencionan capacidades sólo de instrucción; 3) Grupos que mencionan capacidades cognitivas y del contenido; 4) Grupos que mencionan capacidades de instrucción junto con capacidades de contenido; 5) Grupos que mencionan capacidades cognitivas y de instrucción; 6) Grupos que mencionan capacidades que apuntan a los tres aspectos, siendo está la que cubre la definición de capacidad que hemos usado.

Veáse que la Tabla 1 muestra los grupos de cada una de las coincidencias. Esta tabla confirma la tendencia de los futuros maestros de primaria a priorizar en la formulación de las metas capacidades que apunten a los aspectos cognitivos (77\% del total de los grupos) y de instrucción (69\% del total de los grupos). También se puede observar que once grupos hacen mención a estos dos tipos de verbos. La segunda mayor frecuencia (10) son los grupos que utilizan verbos que abarcan todas las componentes de la capacidad, mencionando verbos cognitivos, verbos que reflejan una acción y verbos que aluden al contenido específico. La meta del grupo g1 dice:

[...] Que los alumnos aprendan el concepto de fracción y su representación gráfica y simbólica, asi como a calcular las equivalentes de una fracción dada. Que aprendan a representar fracciones con materiales dados.

(Grupo 1, 2013/2014).

Esta situación ejemplifica la utilización del verbo aprender como capacidad cognitiva, el verbo representar como capacidad del contenido específico de fracciones y calcular como capacidad de acción.

\subsubsection{Variable Contenido}

Como se explicó anteriormente, en esta variable tomamos en cuenta las tres dimensiones de un contenido matemático: estructura conceptual de fracciones, sistema de representación y fenomenología. Separamos estas dimensiones para tener una estructura más detallada de cada meta, con lo que podemos establecer qué elementos de significado de los contenidos matemáticos se mencionan. El análisis se resume en la Tabla 2, que muestra la frecuencia de cada una de las dimensiones. Es importante destacar que los totales que aparecen en el contenido matemático y sistema de representación no tienen correspondencia con el total de los grupos analizados, puesto que un mismo grupo podía hacer referencia a varios. 
Tabla 2 - Variables de especificidad conceptual. Términos claves y frecuencias

\begin{tabular}{|c|c|c|}
\hline Contenidos matemáticos & Sistemas de representación & Sentidos y modos de uso \\
\hline Fracciones: 25 & \multirow[t]{2}{*}{ No indican: 18} & \multirow[t]{2}{*}{ No menciona: 14} \\
\hline Comparar fracciones: 13 & & \\
\hline Representar fracciones: 12 & Físico: 7 & \multirow[t]{2}{*}{ Parte/todo: 20} \\
\hline Números fraccionarios: 9 & Gráfico: 9 & \\
\hline Proporcionalidad: 9 & Simbólico: 11 & \multirow{6}{*}{ Cociente: 1} \\
\hline División igualitaria: 7 & \multirow[t]{5}{*}{ Verbal: 10} & \\
\hline Fraccionamiento: 6 & & \\
\hline Elementos de la fracción: 6 & & \\
\hline Números decimales: 2 & & \\
\hline Redondear: 1 & & \\
\hline $\begin{array}{l}\text { Total: Todos los grupos citan } \\
\text { hasta } 10 \text { elementos de la } \\
\text { estructura de contenido }\end{array}$ & $\begin{array}{c}\text { Total: } 17 \text { grupos citan SR y } 18 \\
\text { no citan SR }\end{array}$ & $\begin{array}{c}\text { Total: } 21 \text { grupos mencionan y } \\
14 \text { no }\end{array}$ \\
\hline
\end{tabular}

Fuente: elaborado por los autores

A continuación, destacamos algunos aspectos de cada una de las dimensiones.

Todos los grupos citan contenidos en sus objetivos. El $71 \%$ de grupos especifican la fracción, le siguen comparación de fracciones y representar fracciones. Menos grupos hacen mención a otros conceptos matemáticos como: proporcionalidad (9) y división igualitaria (7).

Casi la mitad de los grupos hace referencia a algún sistema de representación, teniendo más presencia el simbólico y verbal (una de las actividades de la tarea requiere colocar nombre a porciones de tablas, al hacer el catálogo). Casi un cuarto aluden al sistema gráfico, próximo al físico, pese a que la base de la tarea es la partición física del papel, y no se pide, explícitamente, su representación mediante gráficos.

La mayoría los grupos hacen mención en sus metas al sentido de la fracción parte/todo, que los expertos reconocen como un sentido que utiliza la tarea bricolaje, al emplear una unidad patrón de la que se tienen que obtener partes para hacer tablas de marquetería. Pero una cantidad no menor $(29 \%)$ no mencionan el sentido.

Con respecto a estos últimos tres aspectos apreciamos ciertas coincidencias. La totalidad de los grupos mencionan al menos un contenido, pero, a su vez, algunos grupos hacen mención a los sistemas de representación y al sentido. Esta situación nos llevó a agrupar las coincidencias de cada grupo en referencia a los elementos del significado del contenido que hacen mención en las metas, obteniendo los resultados que se muestran en la Tabla 3.

Tabla 3 - Agrupaciones de respuesta según componentes de significado

\begin{tabular}{llr}
\hline \multicolumn{1}{c}{ Componentes del contenido } & \multicolumn{1}{c}{ Respuestas de grupos } & Frecuencia \\
\hline Contenido, sistema representación y sentido & G5, G8, G11, G12, G13, G15, g6, g7, g8, g9, g10, & $11(31 \%)$ \\
Contenido y sistema representación & G10, g1, g2, g11, g12, g14, g15 & $7(20 \%)$ \\
Contenido y sentido & G1, G4, G7, G9, G14, G17, g5, g13, g16, g17 & $10(29 \%)$ \\
Contenido & G2, G3, G6, G16, G18, g3, g4, & $7(20 \%)$ \\
\hline
\end{tabular}

Fuente: elaborado por los autores

El $31 \%$ de los grupos hacen mención a los tres componentes del significado de un 
concepto, estableciendo uno o varios contenidos matemáticos, uno o varios sistemas de representación y el sentido con que se va a trabajar dicho concepto matemático.

En segundo lugar, diez grupos mencionan uno o varios contenidos y expresan el sentido, dejando de lado los sistemas de representación.

\subsubsection{Variable Situaciones}

La situación en que los futuros maestros enmarcan sus metas, basándonos en las categorías del estudio PISA obtiene los resultados que muestra la Tabla 4.

Tabla 4 - Frecuencia de respuestas en cuanto a la situación

\begin{tabular}{llc}
\hline Situaciones & \multicolumn{1}{c}{ Grupos } & Frecuencia \\
\hline No aluden & G1, G2, G3, G4, G7, G8, G9, G10, G11, G12, G16, G18, g1, g3, g6, g8, g11, & $19(54,3 \%)$ \\
& g14, g16 & \\
Personal & G6, G13, G17, g2, g4, g5, g7, g9, g10, g13, g15 & $11(31,4 \%)$ \\
Laboral & G5, G14, G15, g12, g17 & $5(14,3 \%)$ \\
\hline & \multicolumn{2}{c}{ Total } \\
\hline
\end{tabular}

Fuente: elaborado por los autores

Casi la mitad de los grupos (45,7\%) hacen referencia a situaciones personales y laborales, aquellos que le otorgan una funcionalidad al contenido en la vida cotidiana los enmarcamos en situación personal. Por ejemplo el grupo G6 establece:

[...] los alumnos tienen como expectativas aprender y representar las fracciones en la vida cotidiana.

(Grupo G6, 2015/2016).

La tarea bricolaje está contextualizada en una tienda de bricolaje y el alumno se tiene que situar como trabajador de la tienda, por lo tanto, los cinco grupos que en sus metas hacían alguna relación del contenido con esta situación fueron ubicados la categoría situación laboral. Ejemplo en Figura 4.

- META

Aprender el concepto de fracción como división de un todo. Comprender la proporcionalidad de tamaño respecto al precio, a menor tamaño menor precio.

Figura 4 - Meta del grupo g1.

Fuente: Producciones de los estudiantes

\section{Conclusiones}

La investigación presentada pone de manifiesto que todos los grupos de futuros maestros, en el diseño formativo en el que se realiza el estudio, redactan las metas de una tarea matemática en términos de objetivos de aprendizaje, aunque algunos incorporen finalidades del 
profesor. El resultado de la investigación en relación al primer objetivo (describir los conocimientos didácticos sobre la meta de una tarea matemática escolar que ponen en juego futuros maestros de primaria) estructura el conocimiento didáctico de la meta en tres componentes (cognitiva, de contenido y de instrucción) para las capacidades; el contenido matemático se ha estructurado a su vez en otras tres componentes que atienden al contenido, la representación y el sentido de uso; finalmente, los aspectos de la práctica los hemos considerado desde la perspectiva de las situaciones del marco de evaluación PISA.

Los futuros maestros involucrados en este estudio tienen nociones de estos elementos didácticos involucrados cuando plantean la meta de una TME, como son las capacidades, contenidos y situación (contexto). Sin embargo, no todos llegan a una descripción detallada de cada elemento. Un tercio de los estudiantes describen las capacidades aludiendo a las tres componentes mencionadas por Rico y Lupiáñez (2008), la misma proporción construye el elemento didáctico contenido aludiendo a sus tres componentes consideradas y, finalmente, casi la mitad de los futuros maestros plantea una situación que le de funcionalidad a la meta.

Si bien la complejidad de los objetivos formulados por los estudiantes nos hacen ser optimistas, la particularidad del estudio emprendido, que se basa en que los estudiantes tienen que identificar objetivos a partir de una tarea dada, no es fácil de relacionar con los resultados de investigaciones citadas (DELONG; WINTER; YACKEL, 2005; SCHUNK, 1996), quienes se centraban en la formulación de objetivos al planificar.

Se puede apreciar una formulación de la meta más completa que la observada por RuizHidalgo et al. (2017), lo que podemos relacionar con que la tarea matemática escolar que se ha planteado como reactivo para definir un objetivo reúne más componentes de tarea que la propuesta en ese estudio. La mayor riqueza de la tarea de formación planteada en nuestra investigación en relación al estudio de Santo, Ortigão y Aguiar (2014) se ha visto acompañada de una formulación de objetivos con más precisión de sus elementos.

Respecto al segundo objetivo de investigación, comprender la interpretación que le dan a la meta los futuros maestros, podríamos concluir que relacionan la meta como objetivo de aprendizaje, pero el énfasis se lo dan a las capacidades cognitivas que se desarrollarán con la tarea y al contenido que los alumnos deben adquirir. Por lo tanto, aun sin establecer unos perfiles claros, diferenciamos una escasa cantidad de grupos (4) que satisfacen las tres componentes consideradas para la construcción de un objetivo, ya que estos grupos plantean capacidades que apuntan a procesos cognitivos, de contenido y de instrucción, en cuanto al contenido hacen mención a sus elementos (contenido, sistemas de representación y sentido), para finalmente establecer la funcionalidad en una situación (personal o laboral). 
En definitiva, un aporte de este trabajo es la caracterización que hemos realizado sobre el concepto de meta de las tareas matemáticas escolares, en la que los verbos empleados apuntan al desarrollo de capacidades cognitivas, de acción (instrucción) o bien a capacidades específicas del contenido. Además, incluye la presencia de elementos de contenido como conceptos, sistemas de representación y sentido de uso y, finalmente, la presencia en la meta de situaciones que aportan sentido a los contenidos matemáticos.

\section{Agradecimientos}

Beca CONICYT PFCHA 72150396. Grupo FQM193, Didáctica de la Matemática, Pensamiento Numérico y Proyecto Competencia Didáctica Del Profesor y Aprendizaje de Conceptos Matemáticos Escolares (CODAME).

\section{Referencias}

AGUAYO-ARRIAGADA, C. G. et al. "El bricolaje", tarea para la introducción de las fracciones en el segundo ciclo de Educación Primaria. In: CONGRESO DE ENSEÑANZA Y APRENDIZAJE DE LAS MATEMÁTICAS, 15, 2014, Baeza. Actas... Baeza: SAEM THALES, 2014. p. 177-185.

ARBAUGH, F.; BROWN, C. Analyzing mathematical tasks: a catalyst for change. Journal of Mathematics Teacher Education, Netherlands, v. 8, n. 6, p. 499-536, dic. 2005.

COHEN, L.; MANION, L.; MORRISON, K. Research methods in education. London: Routledge, 2011.

DELONG, M.; WINTER, D.; YACKEL, C. Student learning objectives and mathematics teaching. PRIMUS, Filadeldia, v. 15, n. 3, p. 226-258, 2005.

DOYLE, W. Work in mathematics classes: The context of students' thinking during instruction. Educational Psychologist, Hillsdale, v. 23, n. 2, p. 167-180, 1988.

FERNÁNDEZ PERÉZ, M.; GIMENO, J.; ZABALZA, M. Didáctica II. Madrid: UNED, 1977. FERREIRA, P. E. A.; BURIASCO, R. L. C. Enunciados de tarefas de matemática baseados na perspectiva da Educaçao Matemática Realística. BOLEMA, Rio Claro, v. 29, n. 52, p. 452-472, 2015.

FLORES, P. ¿Por qué multiplicar en cruz? Curso de formación inicial de profesores de matemáticas en la universidad. Conferencia plenaria en: CONGRESO IBEROAMERICANO DE EDUCACIÓN MATEMÁTICA, 7., 2013, Montevideo. Actas... Montevideo, 2013.

FLORES, P.; LUPIÁÑEZ, J. L. Expectativas de aprendizaje. In: RICO, L.; MORENO, A. (Ogr.) Elementos de didáctica de la matemática para el profesor de Secundaria. Madrid: Pirámide, 2016. p. $177-189$.

GÓMEZ, P.; ROMERO, I. Enseñar las matemáticas escolares. In: FLORES, P.; RICO, L. (Org.). Enseñanza y aprendizaje de las matemáticas en Educación Primaria. Madrid: Pirámide, 2015. p. $61-88$.

HERNÁNDEZ, R., FERNÁNDEZ C.; BAPTISTA, P. Metodología de la investigación. México: 
McGraw-Hill, 2010.

KIEREN, T. E. The rational number construct - its elements and mechanisms. In: KIEREN, T. (Org.). Recent Research on Number Learning. Columbus: Eric/Smeac, 1980. p. 125-150

LILJEDAHL, P.; CHERNOFF, E.; ZASKIS, R. Interweaving mathematics and pedagogy in task design: a tale of one task. Journal of Mathematics Teacher Education, Dordrecht, v. 10, n. 6, p. 239249, dec. 2007.

MORENO, A.; RAMÍREZ-UCLÉS, R. Variables y funciones de las tareas matemáticas. In: RICO, L.; MORENO (Ogrs.) Elementos de didáctica de la matemática para el profesor de Secundaria. Madrid: Pirámide, 2016. p. 243-254.

PÉREZ-GARCÍA, P. Metas y propósitos educativos. In: MORAL, C. (Org.). Didáctica. Teoría y práctica de la enseñanza. Madrid: Pirámide, 2010. p. 77-89.

PONTE, J. P.; CHAPMAN, O. Preservice mathematics teachers' knowledge and development. In: ENGLISH, L.D. (Org.). Handbook of international research in mathematics education. New York, NY: Routledge, 2008. p. 223-261.

RICO, L. Bases teóricas del currículo de matemáticas en educación secundaria. Madrid: Síntesis, 1997a.

RICO, L. La enseñanza de las matemáticas en la educación secundaria. Barcelona: Horsori, 1997b.

RICO, L. Matemáticas escolares y conocimiento didáctico. In: FLORES, P.; RICO, L. (Org.).

Enseñanza y aprendizaje de las matemáticas en Educación Primaria. Madrid: Pirámide, 2015. p. $21-40$.

RICO, L. Matemáticas y análisis didáctico. In: RICO, L.; MORENO, A. (Org.). Elementos de didáctica de la matemática para el profesor de Secundaria. Madrid: Pirámide, 2016. p. 85-99.

RICO, L.; LUPIÁÑEZ, J. L. Competencias matemáticas desde una perspectiva curricular. Madrid: Alianza Editorial, 2008.

RUIZ-HIDALGO, J. F. Sentidos y modo de uso de un concepto. In: RICO, L.; MORENO, A. (Org.). Elementos de didáctica de la matemática para el profesor de Secundaria. Madrid: Pirámide, 2016. p. $139-152$.

RUIZ-HILDALGO, J. F. et al. Conocimiento didáctico de maestros en formación sobre objetivos de aprendizaje. In: MUÑOZ-ESCOLANO, J. M. et al. (Org.). Investigación en Educación Matemática XXI. Zaragoza: SEIEM, 2017. p. 437-446.

SANTOS, M. C.; ORTIGÃO, M. I. R.; AGUIAR, G. S. Construção do Currículo de Matemática: como os professores dos anos iniciais compreendem o que deve ser ensinado? BOLEMA, Rio Claro, v. 28, n 49, p. 638-661, 2014.

SCHUNK, D. Goal and self-evaluative influences during children's cognitive skill learning. American Educational Research Journal, California, v 33, n. 2, p. 359-382, 1996.

SHIMIZU, Y. et al. Mathematical task in classroom around the world. Rotterdam: Sense Publishers, 2010.

SHULMAN, L. S. Those who understand: Knowledge growth in teaching. Educational Researcher. Washington, v. 15, n. 2, p. 2-14, feb. 1986. 
STEIN, M. K.; SMITH, M. S. Mathematical tasks as a framework for reflection: from research to practice. Mathematics Teaching in the Middle School, Reston, VA, EUA, v. 3, n. 4, p. 268-275, ene. 1998.

SULLIVAN, P.; CLARKE, D.; CLARKE, B. Teaching with Tasks for Effective Mathematics Learning. New York, NY: Springer, 2013.

SULLIVAN, P. et al. Exploring the relationship between task, teacher actions, and student learning. PNA, Granada, v. 4, n. 4, p. 133-142, jun. 2010.

SULLIVAN, P.; WOODS, T. International Handbook of Mathematics Teacher Education: knowledge and beliefs in mathematics teaching and teaching development. Rotterdam: Sense Publishers, 2008. v. 1.

TIROSH, D.; WOODS, T. The international handbook of mathematics teacher education. Rotterdam: Sense Publishers, 2008. v. 2.

UNIVERSIDAD DE GRANADA. Plan de estudio Grado en Educación Primaria. Guía docente de la asignatura Diseño y desarrollo del currículo de matemáticas en educación primaria. Granada: Autor, 2014.

WATSON, A. et al. Introduction. In: MARGOLINAS, C. Task Design in Mathematics Education. Proceedings of ICMI Study 22. Oxford: ICMI studies, 2013. p. 9-15.

WATSON, A.; MASON, J. Taken-as-shared: a review of common assumptions about mathematical tasks in teacher education. Journal of Mathematics Teacher Education, Londres, v. 10, n. 4-6, p. 205-215, 2007.

Submetido em 11 de Novembro de 2017. Aprovado em 13 de Abril de 2018. 


\section{Anexo 1 - Tarea matemática escolar El Bricolaje}

Cada pareja de alumnos va a poner una tienda de bricolaje, en la que cortan y venden tablas de marquetería. La tabla unidad es un patrón dado (medio A4). Pero también se tienen divisiones de esta tabla patrón.

1) Obtener divisiones que se pueden hacer de la tabla, para tener dispuestas para la venta.

2) Organizar estas tablas en un catálogo, en el que se peguen, se ponga un nombre a cada porción y se indique la razón de tal nombre.

3) Un comprador ha llegado a la carpintería con una tabla como la de la figura. Encontrar qué porción representa, si es igual a alguna de las del catálogo. Si da ideas para nuevas divisiones, completar el catálogo.

4) Fraccionar: Completar el catálogo colocando precios a cada porción, si la tabla patrón cuesta $5 €$.

5) Contraste de catálogos: Compartir los catálogos con otra pareja compañera, intercambiándolos. Compararlos y estudiar qué aportes realiza cada cual

6) Puesta en común: Un grupo describe las diferencias que ha encontrado en los catálogos que ha visto (el propio y el de los compañeros). Se analizan las cualidades de cada catálogo, atendiendo a:

a. Diversidad de porciones representadas y precisión en sus medidas

b. Forma de representar las porciones

c. Precios atribuidos

Finalmente se recuerda la notación fraccionaria para expresar las porciones, y se formaliza los nombres de numerador y denominador

7) Compradores: Va a cambiar su papel a la de compradores. Cada pareja tiene que realizar una caja para minerales, en la que, al menos, haya 6 huecos iguales (figura adjunta). Dibujar la caja que van a construir, señalando sus dimensiones. Seleccionar las tablas necesarias para realizar la caja, expresarlos en un papel por medio de su representación fraccionaria. Redactar una carta escrita, sin dibujos, para pedir los trozos que se necesitan para hacer la caja.

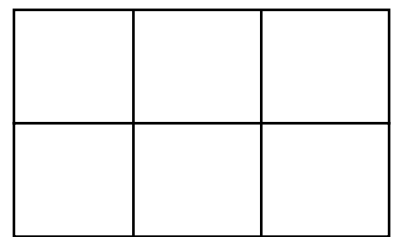

Vista desde arriba

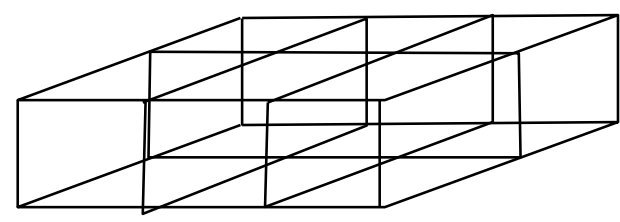

